\title{
Estrategias de conciliación trabajo productivo y reproductivo en parejas de doble ingreso de la ciudad de Talca, Chile
}

\author{
Work-life balance strategies for productive and reproductive \\ work in double-income couples from the city of Talca, Chile
}

\author{
Màrius Domínguez-Amorós \\ mariusdominguez@ub.edu \\ Departamento de Sociología. Universidad de \\ Barcelona, España
}

\section{Verónica Aravena Veja}

veronica.aravena.v@gmail.com

Universidad de Barcelona, España

Recepción: 15 Mayo 2020

Aprobación: 30 Octubre 2020

Publicación: 01 Febrero 2021

Cita sugerida: Domínguez-Amorós, M. y Aravena Veja, V. (2021). Estrategias de conciliación trabajo productivo y reproductivo en parejas de doble ingreso de la ciudad de Talca, Chile. Cuestiones de Sociología, 24, e113. https://doi.org/10.24215/23468904e113
Resumen: En un escenario de intensas transformaciones sociales y familiares, y de búsqueda de equilibrio en la distribución de roles al interior de las familias, la conciliación del trabajo productivo y reproductivo emerge como un elemento central en el desarrollo social de los países. Este estudio busca contribuir a dilucidar los tipos de estrategias de conciliación del trabajo productivoreproductivo, (según presencia o no de hijos/as y algunas variables socio demográficas de interés) utilizadas por parejas de doble ingreso de la ciudad de Talca (Chile), para conciliar ambas esferas y su posible relación con variables sociodemográficas como género, grupo socioeconómico o nivel educacional. La metodología incluyó entrevistas semiestructuradas, seleccionando a 15 parejas de doble ingreso, menores de 45 años, en las cuales ambos integrantes trabajaran fuera del hogar, con el objetivo de identificar la perspectiva del sujeto estudiado, categorías recurrentes en sus discursos y los motivos de sus actos sobre la base de un marco comparable, pero flexible. A pesar que los principales resultados constatan una mayor participación de los varones en las tareas domésticas, su presencia está marcada por una lógica patriarcal que define su rol desde la dominación en la esfera reproductiva.

Palabras clave: Corresponsabilidad, Conciliación, Trabajo Productivo, Trabajo Reproductivo, Roles de Género.

Abstract: In a scenario of intense social and family
transformations, and the search for balance in the distribution of
roles within families, the productive-reproductive work balance
emerges as a central aspect in the social development of the
countries. This study seeks to contribute to elucidating the
types of strategies for reconciling productive-reproductive work,
according to the presence or absence of children and some socio-
demographic variables of interest, used by double-income couples
in the city of Talca (Chile), to worl-life balance and its possible
relationship with socio-demographic variables such as gender,
socioeconomic group or educational level. The methodology
included semi-structured interviews, selecting 15 double-income
couples, under 45 years of age, in which both members worked
outside the home, in order to identify the perspective of the 
subject studied, their mental categories and the reasons for their acts on the basis of a comparable, but flexible framework. Despite the fact that the main results confirm a greater participation of men in domestic tasks, their presence is marked by a patriarchal logic that defines their role from domination in the reproductive sphere.

Keywords: Work, Life Balance, Conciliation, Productive Work, Reproductive Work, Gender Roles.

\section{Introducción}

El presente artículo ${ }^{1}$ analiza el concepto de corresponsabilidad, el cual implica la orquestación de tareas "productivas" y "reproductivas" desde una perspectiva que concilie los espacios de la vida personal, trabajo reproductivo y trabajo productivo de una forma más equitativa entre hombres y mujeres como un elemento crucial para el desarrollo social de los países. Chile, como la mayoría de los países occidentales, ha experimentado la creciente incorporación de las mujeres al empleo remunerado, lo cual ha significado que los sistemas de cuidado tradicionales, basados en el trabajo femenino no remunerado, resultaran insuficientes para satisfacer las expectativas de cuidado y equidad de género. Por ello, incluir el examen de las relaciones de trabajo productivo y reproductivo en el diseño de políticas sociales como parte de un sistema integral nos parece indispensable para asegurar los derechos presentes y futuros del conjunto de la ciudadanía (Jiménez y Moyano, 2008).

Para abordar dicho propósito, el trabajo busca contribuir a dilucidar las diversas estrategias empleadas por parejas de doble ingreso de la ciudad de Talca, Chile, para responder a las demandas del trabajo productivo y reproductivo desde un enfoque cualitativo. Talca resulta una localización pertinente por estar históricamente caracterizada por bajos niveles de desarrollo social, altos niveles de pobreza, envejecimiento demográfico, ruralidad (Ministerio de Desarrollo Social, 2017) y tradicionalismo propio de las ciudades intermedias.

En este sentido, el manuscrito se pregunta sobre las transformaciones efectivas en los roles tradicionales de género: ¿Hasta qué punto, a pesar de veinte años de discurso público de igualdad de género, estas transformaciones han logrado, o no, permear las prácticas concretas de negociación y distribución de trabajo doméstico y de cuidados en la cotidianeidad de los sujetos? Por ello, la hipótesis central del trabajo es que hasta que no se sitúe la corresponsabilidad en el trabajo reproductivo en el plano de una contribución social de relevancia no será posible que se logre un estatus que busque ser compartido.

El artículo está organizado en tres apartados. Primero, hacemos una breve revisión de los cambios sociales ocurridos en Chile en los últimos años, con el objetivo de situar el escenario de la discusión. Luego abordamos los conceptos de corresponsabilidad y trabajo productivo-reproductivo. Posterior a eso se mencionan los principales resultados, y, finalmente, ofrecemos algunas conclusiones organizadas a partir de nuestros argumentos principales. 


\section{Contexto social}

En Chile ha habido un crecimiento económico sostenido en los últimos años, pero, simultáneamente, se observa un marcado deterioro en la calidad de vida de las personas (Martínez, 2008, Otárola, 2007). Dentro de los cambios más importantes experimentados en Chile se observa un engranaje de cambios políticos, económicos y sociales, dentro de los que destacan un sostenido aumento de la participación femenina en el ámbito productivo, el aumento de las expectativas de vida de la población, las demandas de los trabajadores(as) por mejores condiciones de vida, cambios en las relaciones familiares, vertiginosos cambios tecnológicos y organizacionales, entre los más relevantes.

Posiblemente uno de los mayores cambios ha sido el considerable aumento de la participación laboral femenina. Esta inserción se ha explicado principalmente como una forma de apoyar el sustento familiar ayudando a satisfacer necesidades básicas, o como medio de superación de pobreza (LYD, 2006). Se hace habitual que las familias requieran de más de un salario para sustentarse, ya sea por la informalidad y precariedad de los trabajos, o bien por los cambios en patrones de consumo y estilos de vida que generan nuevas necesidades.

En Chile, la inserción laboral de las mujeres se ha dado con menor intensidad -48,9 \% en 2017 (Ministerio de Desarrollo Social, 2017)-, con una brecha de 12 puntos porcentuales respecto a países con un nivel de desarrollo similar (OIT, 2018). La participación laboral femenina favorece la generación de riqueza, aumentando el bienestar de los hogares y disminuyendo la pobreza. No obstante, según la Encuesta de Caracterización Socioeconómica Nacional (CASEN, 2017), entre las mujeres que no están trabajando o buscando trabajo, casi el $62 \%$ indicó razones vinculadas al trabajo doméstico o al cuidado de dependientes, mientras solo $13,7 \%$ de los varones señaló este tipo de razones. Según los datos, las mujeres trabajan menos horas y dejan sus trabajos por razones vinculadas a sus responsabilidades familiares en proporciones significativamente mayores que los varones (Selamé, 2004, Schkolnik, M, 2004).

Estas cifras son todavía más importantes al considerar otros cambios familiares, como que el 73,4 \% de los hogares monoparentales tienen como jefa a una mujer. Las cifras de participación laboral varían al considerarse el nivel de ingreso: mientras en el $10 \%$ de mayor ingreso de la población un 68,2\% de las mujeres participa en el mercado laboral, en el decil de menores recursos esa proporción llega al 30 \% (Ministerio de Desarrollo Social, 2017). Ello da cuenta de la progresiva -y desigual- incorporación de las mujeres al ámbito laboral. A su vez, y todavía según la CASEN, son las mujeres de los segmentos menos favorecidos, las que más plantean la dificultad de encontrar trabajo por responsabilidades específicamente asociadas al cuidado (14,4\% en el primer quintil, versus $4,6 \%$ en el quinto). Las investigaciones realizadas en Chile sugieren que ello se debe a estrategias diferenciadas según clase y circunstancias personales: mientras las mujeres de los segmentos más acomodados pueden transferir estas responsabilidades, total o parcialmente, al mercado (usualmente contratando a otra mujer), las de menos recursos deben apoyarse en redes familiares (Undurraga, 2013).

Las mujeres, al insertarse en el empleo remunerado, todavía deben cumplir las expectativas inherentes a los roles tradicionales asociados al género, sin importar 
si su pareja (cuando está presente en el hogar) tiene o no responsabilidades laborales (Doña, 2007; PNUD, 2018). Las mujeres chilenas dedican 3 horas más que los hombres a las labores domésticas y al cuidado de la familia, lo que aumenta en presencia de niños y adultos mayores en el hogar (2,74 vs. 5,89 horas) (Encuesta Nacional de Uso del Tiempo, 2015). En el caso de la población de entre 25 a 45 años el tiempo dedicado a tareas reproductivas sube en ambos casos, pero la distancia aumenta: los hombres destinan 3,23 horas diarias a estas labores y las mujeres 7,39 (casi cuatro horas de diferencia). Las responsabilidades de los roles tradicionales sumadas a las demandas del trabajo se tensionan entre sí, y condicionan su desarrollo profesional (Chinchilla, 2003).

Lo anterior tiene importantes implicancias desde el punto de vista de la equidad de género, y desde la perspectiva de las oportunidades que las personas en situación de dependencia tienen de recibir cuidado de calidad, en tanto la expectativa social siga asignando esta responsabilidad a mujeres que, en números crecientes, desean y necesitan insertarse en el mercado laboral, afectando tanto a quienes proveen cuidado como a quienes lo reciben -incluidos los niños(as)-, en un contexto marcado por la crisis de cuidado (Arriagada, 2013 y Arriagada y Todaro, 2012).

A pesar de los cambios en los roles femeninos, en materia de trabajo doméstico y de cuidado los hombres chilenos no han modificado sustantivamente su participación. Estudios sugieren que hay interés en los varones jóvenes por ser participantes activos en la crianza de sus hijos (Aguayo, Correa y Cristi, 2011; JUNJI/UNESCO/Unicef, 2010; PNUD, 2010), aunque dicho interés no se traduce necesariamente en una mayor participación de los hombres en el trabajo doméstico y de cuidado, particularmente en lo que se refiere a actividades como atender niños enfermos, cocinar y lavar para ellos (Olavarría, 2004; JUNJI/ UNESCO/Unicef, 2010).

La Encuesta Nacional de la Primera Infancia (ENPI), de 2010, aporta otro dato interesante en este punto: la capacidad diferenciada que hombres y mujeres tienen de negociar sus respuestas a los requerimientos familiares en los espacios laborales. Según esta encuesta, el $91 \%$ de los empleadores respetan el permiso postnatal $^{2}$ de las mujeres; sin embargo, ello ocurre en mayor medida para los niveles socioeconómicos más altos. Entre los hogares de mayores ingresos (estrato $\mathrm{ABC} 1$ en Chile), el $98 \%$ de los empleadores respetan el permiso postnatal, mientras que entre los hogares de menores ingresos (estrato E) solo el $80 \%$ lo hace. En el caso de los padres (varones), se reporta que solo el $55 \%$ de los empleadores autoriza el permiso legal. Nuevamente se repite la relación entre el nivel socioeconómico y cumplimiento legal, ya que entre los padres de los hogares $\mathrm{ABC} 1,72 \%$ hace uso de este derecho; por el contrario, solo el $34 \%$ de los padres de los hogares de NSE (Nivel socioeconómico) E reporta haber podido utilizarlo (JUNI/UNESCO/Unicef, 2010, p. 38). Esta misma tendencia se verificaría en la utilización de otros derechos y beneficios.

Así, los datos disponibles sugieren la persistencia de los patrones culturales tradicionales en la familia: si bien los varones muestran interés en pasar tiempo con sus hijos, las tareas tradicionalmente asociadas al cuidado siguen siendo predominantemente realizadas por mujeres. Es igualmente importante que en el ámbito del trabajo habría diferencias en el respeto a derechos ya consagrados por sexo y por nivel socioeconómico, lo cual da cuenta de cómo las obligaciones 
laborales y familiares aún se ven en tensión socialmente. Si bien desde el punto de vista cultural se observan cambios en el sentido de la maternidad y paternidad, así como en la construcción de roles y responsabilidades asociados a la crianza -siendo cada vez más legitimada una estructura de roles compartidos-, las percepciones, actitudes y comportamientos frente a la mujer y su rol muestran fuertes contradicciones, en las que coexisten aspectos tradicionales y progresistas (SERNAM, 2002).

Así pues, si se plantea una visión comparativa de las normas culturales mediante indicadores referidos a la ideología y a las actitudes hacia los roles de género y los estereotipos- a partir de la Encuesta Mundial sobre Valores Sociales (Institute for Comparative Survey Research, 2017) (ver tabla 1), veremos que en Chile en un contexto de escasos puestos ocupacionales en el mercado laboral, los varones deben tener más derecho a un trabajo (41,9\%), y que si la mujer gana más que el varón esto puede traer ciertos problemas $(66,2 \%)$.

Tabla 1

Opiniones sobre aspectos relacionados con la ideología de género, por país. Encuesta de valores mundiales (porcentaje de respuestas que expresan acuerdo con la declaración o neutralidad).

\begin{tabular}{|c|c|c|c|c|c|c|c|c|c|c|}
\hline & $\begin{array}{l}\frac{\pi}{0} \\
\frac{\pi}{2} \\
\frac{\pi}{4}\end{array}$ & 究 & $\stackrel{\frac{\pi}{\pi}}{\underline{\pi}}$ & 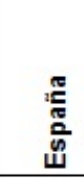 & 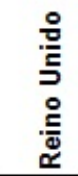 & : & 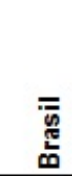 & $\frac{\stackrel{5}{\frac{5}{0}}}{}$ & 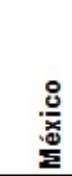 & 촐 \\
\hline $\begin{array}{l}\text { Cuando hay poco } \\
\text { empleo, los hombres } \\
\text { deben tener más } \\
\text { derecho a un trabajo }\end{array}$ & 26.2 & 18.4 & 39.8 & 17.5 & 23.3 & 29.5 & 26.8 & 41.9 & 29.3 & 32.5 \\
\hline $\begin{array}{l}\text { Si una mujer gana más } \\
\text { que su esposo, esto } \\
\text { seguramente creará } \\
\text { problemas }\end{array}$ & - & - & - & 25.0 & - & 46.0 & 50.5 & 66.2 & 57.3 & 38.6 \\
\hline $\begin{array}{l}\text { Cuando una madre } \\
\text { tiene un trabajo } \\
\text { remunerado, sus hijos } \\
\text { sufren }\end{array}$ & - & - & - & 28.5 & - & - & 60.4 & 37.8 & 43.6 & 37.4 \\
\hline $\begin{array}{l}\text { En general, los } \\
\text { hombres son mejores } \\
\text { líderes políticos que las } \\
\text { mujeres. }\end{array}$ & 20.7 & 18.7 & 17.9 & 11.5 & 17.8 & 27.5 & 28.4 & 28.2 & 23.0 & 9.1 \\
\hline $\begin{array}{l}\text { Una educación } \\
\text { universitaria es más } \\
\text { importante para un niño } \\
\text { que para una niña }\end{array}$ & 6.7 & 6.3 & 7.8 & 11.7 & 6.6 & 16.6 & 9.4 & 20.9 & 20.7 & 9.7 \\
\hline $\begin{array}{l}\text { Ser ama de casa es } \\
\text { casi tan satisfactorio } \\
\text { como tener un trabajo } \\
\text { remunerado }\end{array}$ & 50.0 & 82.0 & 49.8 & 49.5 & 59.7 & 54.2 & 44.4 & 44.4 & 58.2 & 59.7 \\
\hline
\end{tabular}

Domínguez, M.; Muñiz, L.; Rubilar, G. (2020), a partir de la sexta oleada de las Encuestas de Valores Mundiales. Argentina, 2013; Brasil, 2014; Chile, 2012; Finlandia, 2005; Francia, 2006; Italia, 2005; México, 2012; España, 2011; Reino Unido, 2005; y Uruguay, 2011.

Considerando lo anterior, este estudio busca caracterizar el proceso de negociación familiar sobre tareas domésticas y de cuidado, con foco en las eventuales estrategias utilizadas por parejas de doble ingreso de la ciudad de Talca, para conciliar el trabajo productivo y reproductivo, y su posible relación con variables sociodemográficas como género, grupo socioeconómico o nivel educacional. 


\section{Trabajo reproductivo y corresponsabilidad}

El trabajo reproductivo incluye tareas domésticas y de cuidado que tienden a superponerse entre sí. El propio concepto no está exento de debate, con distintas conceptualizaciones y aproximaciones (Domínguez et al., 2019), definido ampliamente por María ngeles Durán (2018) como todo el tiempo dedicado en el hogar a la familia y al propio autocuidado. Este trabajo no ocurre solo en el hogar, aunque se enfoca principalmente a satisfacer las necesidades del grupo familiar e implica no solo actividades reproductivas (como dar a luz y criar un bebé), sino también tareas domésticas. Se le denomina así para diferenciarlo del trabajo "productivo" (de bienes y servicios), que en las sociedades industrializadas es generalmente el único reconocido, económica y socialmente, como trabajo (Prieto, 2015).

De forma concreta, se constata como actividades reproductivas, aquellas realizadas sin remuneración desde los hogares, siendo así uno de los componentes fundamentales del bienestar social, y realizado fundamentalmente por las mujeres, en un contexto de substitución del modelo más tradicional de un solo proveedor malebreadwinner/housewife household) por el modelo de dos preceptores de ingresos (Díaz y Simó, 2016; Díaz et al., 2015; Finkel, 1997).

El cuidado en sí mismo es una fuente inacabable de bienestar social, que de manera privada y con base en el hogar está convenientemente invisibilizado por el sistema capitalista actual, por esta razón, su coste es trasladado a las familias y principalmente a las mujeres, quienes, según Durán (2018), conforman una nueva clase social a la que define como cuidatoriado. Esta clase social comparte con el proletariado ejes centrales; subordinación, nula protección social, riesgo de pobreza, opresión y marginación, con alguna característica propia mediada por la perspectiva de género.

En este sentido, la búsqueda del equilibrio del trabajo productivoreproductivo debiera ser parte integral de una estrategia que busca asegurar a niños, niñas y personas dependientes en general un cuidado de calidad, considerando que las tensiones entre estas dos esferas parecen tener un impacto directo en la percepción de autoeficacia parental ${ }^{3}$ (Farkas, 2008), entre otras consecuencias.

Ello parece ser de particular importancia para las familias de bajos ingresos, ya que la evidencia disponible sugiere que estas familias enfrentan mayores dificultades para asegurar el cumplimiento de sus derechos laborales referidos a paternidad/maternidad (JUNJI/UNESCO/Unicef, 2010).

A la luz de esta información, es posible plantear que el aseguramiento de derechos para los niños(as), particularmente en relación al cuidado integral en las primeras etapas de la vida, está estrechamento relacionada con lo que autoras como Razavi (2007), Lamaute-Brisson (2013) y Arriagada y Todaro (2012) denominan la organización social del cuidado. Como observa Sojo (2011), la regla general es que las preocupaciones gubernamentales asociadas al cuidado sean incipientes y sigan concentradas en las familias, sin considerar un contexto laboral que demanda largas horas de trabajo, permite escasa flexibilidad y provee pocas garantías de protección social.

Así, las políticas de infancia y del adulto mayor se han situado progresivamente dentro de un marco de derechos, pero discurren por los cauces tradicionales, 
carentes de un marco de referencia común en la temática del cuidado (Sojo, 2011, p. 6).

Las políticas públicas en el ámbito del cuidado implican nuevos equilibrios de las interrelaciones entre Estado, mercado y familias, y pueden apuntar a muy diversos objetivos, que podrían retroalimentarse positivamente en el curso del tiempo. En esta misma línea, la literatura sobre conciliación trabajo productivo- reproductivo plantea tres pilares para equilibrar las demandas laborales y familiares: el trabajador, el Estado y las organizaciones/empresas (Debeljuh y Jáuregui, 2004). En cuanto al trabajador, este debe desarrollar e implementar "arreglos domésticos" para buscar armonizar y responder a las demandas familiares y laborales de una manera corresponsable, entendida como una distribución equitativa entre los miembros de la pareja (o de la familia, si ya hay niños en edad de asumir tareas de esta naturaleza) de la energía, tiempo y desarrollo de habilidades que resultan determinantes en una familia, para llevar a cabo las tareas del hogar y el cuidado de los hijos (Torió, Peña y Rodríguez, 2010). Por su parte, el Estado debe velar por los intereses de cada uno de los miembros de la familia, diseñando marcos legales, tales como políticas laborales amigables, que permitan el equilibrio del trabajo productivo-reproductivo. Finalmente se encuentra el mundo del trabajo remunerado, encargado de crear un ambiente laboral que posibilite el equilibrio en ambos dominios para sus trabajadores(as).

Esta tríada implica que el cuidado requiere una reconceptualización de esta tarea como una responsabilidad de la sociedad, y no solo de las familias -o solo de las mujeres- (Domínguez et al., 2019; Durán, 2018; Díaz et al. 2016; Prieto, 2015). Según Pautassi (2010), los debates en la arena internacional avanzan hacia la noción del cuidado como un derecho de las personas, inherente a la igualdad de oportunidades, de trato y de trayectoria. Sin embargo, los arreglos societales necesarios para acompañar la readecuación de la oferta de cuidado, y su distribución dentro de la sociedad de una manera más equitativa, no se han verificado. Según esta autora, las políticas sociales dirigidas a la protección de menores (tales como sistemas educativos para niños y jóvenes o programas de salud materno-infantiles) conciben el cuidado no como eje de la regulación y de las políticas, sino como "herramienta para la inserción de las mujeres en el empleo remunerado, con total prescindencia de la responsabilidad paterna o masculina, o como parte de programas asistenciales en la esfera de ministerios sectoriales" (Pautassi, 2010, p. 72).

Y es que el margen de acción necesario para desarrollar estrategias personales de conciliación laboral y familiar está fuertemente marcado por el género y la clase social, en tanto determinantes sociales del modo en que las personas se insertan en el mundo laboral y las expectativas que acompañan a los roles de género tradicionales. De acuerdo con el estudio del 2013 de la Fundación Chile Unido, que otorga el premio "Mejores empresas para padres y madres que trabajan", existe consenso en los colaboradores de las 114 organizaciones participantes (que incluyen entidades públicas y privadas de diversos tamaños) respecto a que la flexibilidad en el horario de trabajo basada en el cumplimiento de objetivos o trabajar desde la casa era la medida a la que se asignaba mayor prioridad, y, paradójicamente, una de las menos implementadas.

Según Tobío (2002), se ha encontrado que las personas, para lograr superar lo que podría ser una incompatibilidad del trabajo productivo-reproductivo, 
utilizan varios tipos de estrategia: estrategias principales, que son aquellas que resultan suficientes para que se pueda responder a las demandas de la esfera doméstica; allí se encuentran, por ejemplo, el caso en que algún miembro de parentesco femenino actúa como madre sustituta, o en que una persona ajena asume el mencionado rol a cambio de una remuneración económica. Las estrategias complementarias son las que por sí mismas no son suficientes para resolver las dificultades que impiden que se logre una compatibilidad entre la familia y el trabajo remunerado, por lo que deben combinarse con otra estrategia. Las estrategias extremas o indeseables, que son negativas ya sea para la madre o para los hijos, y que continúan existiendo a pesar de ser consideradas dañinas; aquí se encuentran las prácticas como llevar a los hijos al trabajo, no acudir al trabajo cuando los hijos se encuentran enfermos o dejarlos solos en casa aun cuando estos son pequeños. Por último, las estrategias indirectas, a partir de las cuales para lograr la compatibilidad se debe abandonar a una de las partes, ya sea el trabajo remunerado o los hijos.

En general, los datos disponibles sugieren que las parejas desarrollan estrategias conjuntas para aminorar el conflicto posible derivado de la incompatibilidad de ambos dominios. La planificación y decisiones conjuntas en lo que respecta a tareas domésticas y cuidado de dependientes son las estrategias más destacadas por las parejas, lo cual revela la gran importancia que le atribuyen a los consensos.

Sin embargo, se evidencian diferencias por sexo a la hora de conciliar trabajo productivo y reproductivo. En el caso de las mujeres, optan por reajustar su jornada laboral. Otra opción bastante utilizada es recurrir al apoyo interpersonal social, principalmente el cuidado que brindan familiares, especialmente mujeres, lo que viene a reforzar el estereotipo femenino de cuidado, lo que demuestra que la conciliación de estas dos esferas es una responsabilidad exclusiva de las familias, especialmente de las mujeres (Torio, 2010, Román, Avendaño y Tomicic, 2004).

En esta línea, la hipótesis central de este estudio señala que las estrategias finalmente adoptadas para conciliar el trabajo productivo y reproductivo dependen tanto de las representaciones propias de los sujetos como de las oportunidades que el contexto familiar y laboral ofrezca para superar la división tradicional de tareas entre hombres y mujeres. A mayor número de hijos y menor NSE se espera una mayor opción por estrategias de conciliación de tipo tradicional, debido a que este grupo contaría con márgenes de negociación menores.

\section{Método}

Como técnica de recolección de la información se utilizó la entrevista semiestructurada, instrumento que permite acceder a la perspectiva del sujeto estudiado, comprender categorías recurrentes en sus discursos y los motivos de sus actos sobre la base de un marco comparable, pero flexible (Wengraf, 2004). Para esto se seleccionó a 15 parejas de doble ingreso en las cuales ambos integrantes trabajaran fuera del hogar, en una jornada de al menos 22 horas semanales o su equivalente. La selección fue hecha a través de una invitación abierta a la comunidad a participar en el estudio, así como del uso de la estrategia de bola de nieve, para reunir a parejas que cumplieran con los requisitos de ser menores de 45 años (como generación expuesta a cambios en los modelos 
familiares y de protección social en Chile), con hijos(as) o sin ellos. Este último factor básicamente responde a la necesidad de conocer cómo la presencia o ausencia de niños(as) condiciona o no las pautas distributivas del trabajo reproductivo.

En el caso de las parejas con hijos(as), los mismos debían tener hasta 14 años, dado el impacto en la organización del cuidado que tienen los menores en esa etapa. Del grupo seleccionado, seis parejas no tenían hijos(as), en tanto cuatro tenían hijos(as) menores de 6, y las restantes, entre los 6 y los 14 años. Se procuró que las parejas tuvieran al menos dos años de cohabitación (matrimonio o convivencia), y que hubiera una representación equitativa entre profesionales con altos niveles de autonomía y otras ocupaciones con exigencias más rígidas en términos de tareas, horarios y disponibilidad física del trabajador(a) en el lugar de trabajo.

En términos de NSE, se utilizó el nivel educacional como proxy (dada la alta correlación entre nivel educacional y de ingreso en Chile), y se seleccionó, para cada sexo, a ocho personas con educación superior completa (universitaria o técnica-profesional) y a siete bajo ese nivel educativo. Tanto la edad como el nivel educacional son claves, pues los datos disponibles sugieren que hay un cambio generacional en las actitudes de género en los menores de 45 años, y que ese cambio es más acentuado en quienes tienen mayores niveles de educación formal.

Cada miembro de la pareja fue entrevistado(a) por separado, durante los meses de agosto y septiembre del año 2019 en la ciudad de Talca. Las entrevistas se llevaron a cabo mayoritariamente en los domicilios particulares de cada participante; previo a la entrevista se les dio a conocer en qué consistía el estudio, además de los detalles éticos y de confidencialidad correspondientes. Por último, se les solicitó autorización para grabar y tomar notas durante las entrevistas. Terminada esta fase cada uno de los(as) entrevistados(as) firmaron un consentimiento informado. La entrevista contaba con un apartado inicial de variables sociodemográficas, luego con una sección basada en la tipología sobre trabajo doméstico y de cuidado del Programa de Naciones Unidas para el Desarrollo (2010), y, por último, con un apartado sobre representaciones de género.

El método de análisis fue la identificación de categorías recurrentes en el discurso, que dieran cuenta de representaciones sobre trabajo doméstico y de cuidado, y los roles identificados para hombres y mujeres en esta materia, si los hubiera. Estas categorías fueron definidas a priori con los referentes provistos por la teoría de género, aunque se consideraron también categorías emergentes (Pérez, 2004). En una segunda etapa se identificaron relaciones/asociaciones entre categorías, con la finalidad de identificar ideas o argumentos recurrentes para grupos según las variables sociodemográficas definidas, o bien con la de constatar la inexistencia de tales diferencias. Finalmente, las categorías que permeaban todos los discursos fueron agrupadas en lo que se consideró el núcleo figurativo de la representación.

\section{Resultados}

Uno de los hallazgos más llamativos que se pudo constatar es que el rol de los hombres en los espacios no es solo una cuestión de hacer o no hacer. El cómo es 
crucial. Así, si solo consideramos los números, observamos que, efectivamente, muchos hombres participan cada vez más de las tareas domésticas, pero, como se sabe, lo importante se esconde en los detalles. Muchos de los testimonios que son parte de esta investigación hablan de padres o hermanos que si bien participan más de las tareas domésticas, lo hacen de forma dominante, disponiendo e intentando estructurar el espacio sin sopesar si había un orden previo, y asumiendo que sus maneras son más eficaces solo por ser suyas.

Estos varones trasladan lógicas patriarcales a su implicación en lo doméstico, rearticulando de manera híbrida una masculinidad tradicional con una más igualitaria. Estos hombres igualitarios pero patriarcales limpian, cocinan, friegan, pero lo hacen con formas dominantes. Monopolizan el espacio, marcan el ritmo y controlan la logística de las tareas del hogar.

Si bien ninguna(o) de las(os) participantes declara considerar tareas específicas por sexo, en la práctica se constata la inmutación de los patrones tradicionales de reparto, aunque con reformulaciones sutiles (que también responden a los nuevos escenarios y configuraciones familiares).

En línea con lo tradicional y con lo señalado en otras investigaciones (Finkel, 1997), los hombres declaran ocuparse principalmente de lo que ocurre fuera de la casa (mantención del jardín, ir a comprar, pagar cuentas) o en áreas típicamente masculinas: arreglar desperfectos dentro del hogar -cañerías, bombillos, etc.-, sacar la basura, cortar o apilar leña. Y, en un ámbito relativamente nuevo, también declaran asumir nuevas tareas típicamente consideradas femeninas, como cocinar u ocuparse de los niños(as), cuando los hay.

Una vez lo he visto tomar la iniciativa para bañarlo (Hijo), porque yo estaba haciendo mil cosas y la verdad para pelear por más cuestiones no; siento un peso mental, él es como un monigote; si yo le digo lo hace, pero él pocas veces lo hace, para cosas que le gusta sí, para comprar él va, porque le gusta, pero si ve el baño sucio no lo limpia, yo el domingo encero porque es complejo, si le digo a él lo hace, pero me da lata andar diciéndole, prefiero hacerlo yo (G. Sánchez, profesional, con un hijo, comunicación personales, Septiembre, 2019).

La cita arriba ilustra una relación frecuentemente encontrada en las narraciones de los(as) informantes: si bien hombres y mujeres atribuyen importancia a la limpieza del hogar, los varones declaran que tareas tales como limpiar el baño no les resultan agradables, y, por ende, se asume que es la pareja quien debe ocuparse de ello. Cocinar, en cambio, especialmente cuando se hace para invitados fuera del grupo familiar inmediato (barbacoas, asados), es una tarea que los varones sienten que pueden realizar y que deja un producto visible, tangible -una habilidad perceptible para el entorno-. Limpiar, por el contrario, es algo que solo se percibe "cuando está mal hecho", como observará uno de nuestros informantes.

Lo anteriormente descrito no es nuevo. Los varones siempre han estado en la casa. Las teorías que señalan que la validación de la masculinidad solo se da en la dimensión pública pasan por alto numerosa literatura que señala que ciertas tareas de la casa actúan validando lo masculino: el bricolaje, el cuidado del auto, la barbacoa, el culto a las herramientas; es más, se observa casi como una regla que los hombres cocinen los sábados por la noche algo "especial” (Candela y Piñón, 2013). 
En este sentido, podemos decir que los hombres se involucran más en el trabajo doméstico, pero de una forma dominante: señalando qué hacer, buscando protagonismo y recibiendo muy mal la crítica. Es decir, son hombres domésticos, pero con lógicas patriarcales.

Lo anterior es importante porque muestra las condicionantes de la distribución de tareas entre hombres y mujeres; aunque no se declara la creencia en roles de género, esta se manifiesta bajo la forma de la vigencia de la asignación de las tareas domésticas a las mujeres. Ellas son, en último término, las encargadas de organizar la vida cotidiana, mientras ellos asumen un rol más bien subsidiario, que aparece frecuentemente en los discursos masculinos, en expresiones como "ayudar en la casa” o "colaborar con la pareja” (Saldaña, 2018).

$\mathrm{Al}$ consultarse por la forma en que se llegó a la actual distribución de tareas y de recursos financieros, otro patrón de interés emerge: gran parte de las mujeres profesionales (NSE medio alto) hablan explícitamente de una negociación que, en todos los casos excepto en uno, fue iniciada por ellas; esto es, fueron las mujeres quienes plantearon la necesidad de redefinir roles y redistribuir tareas. Esto, en parejas sin hijos. Un punto que se repite con fuerza en las parejas que reportan haber experimentado un proceso de negociación es que ese proceso nace a raíz de una especie de "colapso" de la mujer por la excesiva carga total de trabajo, o por situaciones de crisis, como enfermedad de hijos, o padres/madres adultos mayores. A raíz de aquella situación extrema es que muchas mujeres señalan haber "pedido auxilio" a sus respectivas parejas para replantear la distribución de tareas de ese entonces.

En los demás casos se habla de un proceso que se dio "naturalmente", una distribución de tareas que "fluye" o se habría dado de manera espontánea, en teoría, basada en las preferencias y habilidades de cada uno de los miembros de la pareja. Se puede agregar, además, que las parejas que reportan el reparto de tareas como un proceso natural, generalmente describen dinámicas poco igualitarias, muy alejadas de la corresponsabilidad y generalmente con hijos(as).

No obstante, la descripción de las rutinas cotidianas revela que, se hable de negociación o no, las mujeres reportan que les toca una parcela mayoritaria de las tareas del hogar, ya sea porque los términos de la negociación no se cumplieron (y no se desea volver a la discusión inicial), o porque los varones hacen "lo que pueden" y ellas deben terminar lo que se hizo a medias, con pérdida de tiempo personal. Cabe señalar que los argumentos de la mayoría de los hombres para explicar su baja contribución a las tareas domésticas y de cuidados son la "falta de tiempo", que "no saben", o que "no pueden". En los discursos femeninos se reportan muy pocas referencias a tiempo personal propio -salir con amigos(as), hacer deportes o cultivar pasatiempos-, con la excepción parcial de las mujeres sin hijos(as). Ello no ocurre en el caso de los varones, quienes sí plantean referencias espontáneas a tiempo personal, a participación de grupos sociales, deportivos, utilización del espacio público y de instancias de participación política e intelectual.

Al preguntarle a las parejas con hijos(as) por la distribución de las tareas de cuidado infantil, las referencias a procesos de negociación prácticamente desaparecen, incluso entre quienes reportaron negociar trabajo doméstico y dinero. En consonancia con otros estudios (Finkel, 1997), aquí reaparece el rol materno como preponderante: los hombres reconocen querer ser padres más 
cercanos y activos en la crianza de sus hijos(as), lo cual se traduce en que estén más disponibles para lo lúdico y la comunicación, y, entre quienes tienen hijos(as) en edad escolar, para apoyar la realización de los deberes y tareas del colegio. No obstante, las tareas cotidianas de reproducción de cuerpos - bañar, vestir, cuidar de la ropa- se dejan en manos femeninas, especialmente en caso de enfermedad de un hijo(a), como se muestra en la siguiente cita:

\begin{abstract}
Cuando se ha enfermado, o tiro licencia o pido permiso, o le pido a mi mamá que viaje; yo no lo converso con J (marido), como para mí es la prioridad, yo pido permiso de inmediato. Yo tomo la iniciativa y pido permiso. Yo creo que a él le causa más problema pedir permiso (V. Soto, no profesional, 1 hija menor de 6 años, comunicación personal, septiembre,2019).

Con respecto a mi hijo, en general la contención y el cuidado lo ve un poco más mi señora, esto se ha dado de manera natural; yo no tengo idea qué ropa ocupa. Eso lo ve ella, por desinterés mío también, esto se va dando, el tema de cuidarlo cuando está enfermo es porque mi hijo siente más protección cuando está con la mamá que con el papá, a mí me ve como una figura más de juego, eso es por habilidades y por características de personalidad (Aravena, profesional, hijo menor de 6 años, comunicación personal, Septiembre,2019).
\end{abstract}

Algunos datos de contexto pueden ayudarnos a comprender este fenómeno. El estudio de 2011 realizado por Aguayo, Correa y Cristi (2011) en tres grandes urbes chilenas mostró que 97,8 \% de los hombres considera importante que el padre esté presente en la vida de sus hijos, incluso si está separado de la madre. Sin embargo, 46 \% afirmó que cambiar pañales, bañar y alimentar a los niños es responsabilidad de la madre. Estas representaciones se vinculan con la historia familiar, que fue una categoría recurrente al tratar el tema de la relación con los hijos(as): muchos varones, especialmente de NSE medio-bajo, declararon querer distanciarse de un modelo paterno, cuya prioridad es el trabajo remunerado, que deja poco tiempo y energía para escuchar a los hijos o apoyar sus obligaciones escolares. A pesar de ello, al preguntarles por su respuesta en el caso hipotético de una emergencia, enfermedad o accidente de un hijo(a), la gran mayoría de ellos planteó como estrategia de afrontamiento llamar a la madre o a la abuela del menor (o sea, a una mujer). Las mujeres reforzaron este patrón, ya que todas ellas plantearon que dejarían el trabajo sin pensarlo para acudir a ver al hijo(a) y que "después veo qué hago con mi trabajo", como señala una de nuestras informantes, aunque las redes familiares (madres y abuelas, especialmente) aparecen como un recurso de gran importancia para las mujeres también. El siguiente extracto ilustra esta idea:

\footnotetext{
Yo pido licencia cuando está enfermo (hijo), siempre es así, como yo soy la mamá y aún toma leche es obvio. La mamá tiene que ir a las reuniones según yo, él (pareja) no ha dicho nada de querer participar en reuniones. Es que se ha dado así la distribución, a veces igual le digo ayúdeme, porque me canso y él me dice que él me ayuda, pero casi siempre le pido ayuda a mi mamá o hermana. La mayor carga es mía; a veces le reclamo, porque puede estar toda la tarde y no hace nada, yo le pido ayuda y él dice que ordena... (risas) (I. Barria, no profesional, un hijo menor de 6 años, comunicación personal, septiembre,2019).
}

Aquí es claro que el peso de los roles tradicionales aún se hace sentir, pero también cabe hipotetizar la persistencia de las desigualdades de poder de las que hablamos al inicio de este trabajo. Esto es, las personas no solo sienten (a nivel normativo) que deben cumplir con ciertas obligaciones en función de su sexo, 
sino que también hay márgenes de negociación estrechos o inexistentes. Desde esta perspectiva, si nuestros informantes siguen el patrón existente en Chile, las mujeres recibirán salarios inferiores a los de los hombres, tanto debido a que se concentran en ocupaciones con niveles más bajos de remuneración, como porque, debido a la desigualdad de género, ellas reciben montos inferiores por un mismo trabajo - situación que se agudiza cuanto mayor es el nivel de educación de la mujer (INE, 2013)-. Ello implica que su aporte económico al hogar será menor en la mayoría de los casos. Por ello, las mujeres tendrán menos poder de negociación en el hogar.

En relación con este punto, las parejas sin hijos reportan estar conscientes de que los arreglos familiares actuales experimentarán un cambio radical con el nacimiento de un bebé. En las parejas con niños(as) una estrategia frecuente involucra el recurso a las abuelas o que ella busque una ocupación más flexible o que reduzca, al menos temporalmente, sus horas de trabajo remunerado (regresando a un patrón de género tradicional). Las mismas estrategias aparecen como posibilidad para quienes no tienen hijos(as), como muestra la cita siguiente, representativa de las conversaciones sobre la cuestión de la maternidad en las parejas sin hijos(as):

Una tiene que estar pendiente de todo ser como un radar; hay que captar las
necesidades para poder decir necesitamos esto, hay que hacer esto otro. En mi caso
ya me tienen convencida que la responsabilidad es siempre de la mujer, ya me repiten
esa historia que es la mujer la responsable (su marido se lo dice), sobre todo cuando
hablamos de la posibilidad de tener un hijo. Yo creo que sería más protectora; estaría
más pendiente de los hijos que él y por otra parte también le pediría ayuda a mi mamá
quizás (F. Merino, profesional, sin hijos, comunicación personal, septiembre, 2019).

En esta línea, fueron las mujeres con educación superior y con contrato a tiempo completo las que reportaron mayores posibilidades de negociar sus responsabilidades familiares en el ámbito del trabajo remunerado. Esto también es consistente con otros estudios, que sugieren que las mujeres con mayores niveles de educación (y, por ello, con mejores expectativas laborales) se incorporan en mayor proporción al mercado laboral (Ministerio de Desarrollo Social, 2011). Ello, porque este segmento puede recurrir a la contratación de servicios de terceras. Al mismo tiempo, quienes son más difíciles de reemplazar por parte de las organizaciones suelen tener espacios de autonomía mayores para compatibilizar familia y trabajo remunerado (Gornick y Meyers, 2009). La paradoja radica en que, como muestra la cita arriba, este grupo suele ser el que tiene mayor consciencia de las dificultades implicadas en el proyecto de conciliar familia y trabajo remunerado.

Ninguno de los(as) entrevistados(as) reportó estrategias institucionales -más allá de lo exigido por la ley laboral chilena- para facilitar la conciliación entre responsabilidades laborales y familiares, lo cual supone que las negociaciones que puedan ser llevadas a cabo en lo laboral son altamente dependientes de circunstancias y recursos personales. Un relato frecuente en este sentido tiene que ver con la discrecionalidad de la jefatura y las posibilidades que el tipo de ocupación proporciona para responder a situaciones familiares que requieren la presencia paterna o materna en el horario de trabajo:

Muchas veces he dejado de lado cosas de mi hijo porque en la pega te juzgan de otra forma que a los hombres; el hombre dice voy al doctor con mi hijo y nada y a la mujer 
le dicen otra vez de nuevo, y me pasó harto; de hecho me echaron de una pega por eso, por pedir permiso. Nunca ha sido lo mismo, siempre he notado esa diferencia (A. Cuevas, profesional, 1 hijo menor de 6, comunicación personal, septiembre, 2019).

Cuando alguna de las niñas se enferma (hijas), nos organizamos bien y pedimos días en el trabajo, nos turnamos, siempre y cuando no nos perjudique en el trabajo. Yo por lo general tengo más chance de pedir permiso, porque las horas extras que trabajo me las devuelven en tiempo y puedo echar mano de eso. Pero aun asílo debo negociar con los jefes, depende también del volumen de trabajo. Normalmente pedimos ayuda a los abuelos, o, si no, contratamos a alguien por horas. Mi señora se encarga de buscar a alguien para que cuide a las niñas (R, Vega, no profesional, 2 hijas mayores de 6, comunicación personal, septiembre,2019).

La cita arriba ilustra una idea frecuente: el jefe(a) otorga facilidades para responder a la exigencia familiar, pero la respuesta debe ser organizada de manera personal, particularmente en aquellos trabajos en los que parte de las tareas pueden ser recuperadas en casa o en otro horario. Otros trabajos, con requerimientos horarios mucho menos flexibles, simplemente no ofrecen esa posibilidad, como reportaron quienes tienen ocupaciones consideradas de menor calificación. Y, en estos casos, las estrategias adaptativas de las mujeres involucran reducir su propio tiempo de trabajo (y, con ello, su ingreso monetario), cosa que los varones no hacen.

\section{Conclusiones}

La casa es una de las dimensiones fundamentales para comprender el reparto de poderes de género. "La casa" ha sido teorizada desde diversas perspectivas. El feminismo blanco la ha denominado como uno de los terrenos claves de opresión y encarnación de las desigualdades de género. Feministas decoloniales como Bell Hooks (2017) cuestionan esa idea denominándola como eurocéntrica, y sitúan a la casa como el espacio sagrado alejado de la esclavitud. No obstante, de una perspectiva u otra, "la casa" se presenta siempre condicionada por la distribución de poderes sociales según criterios de género.

En este sentido, si bien se puede constatar una mayor participación de los varones en las tareas domésticas, su presencia está marcada por una lógica patriarcal, que define su rol desde la dominación en la esfera reproductiva. Aunque participen, son conscientes de que desempeñan un rol subsidiario. Incluso en los niveles de mayor NSE, son las mujeres quienes deben buscar las estrategias de conciliación del trabajo productivo y reproductivo, que, por lo demás, es otra herramienta política patriarcal, tímidamente cuestionada, que suma otra carga mental a las mujeres trabajadoras, y pone en ellas la responsabilidad de articular estas dos esferas. Solo como último resorte -cuando no es posible contar con madres, abuelas o vecinas- se recurre a alterar la rutina masculina, lo que muestra nuevamente la poca posibilidad de decidir de las mujeres.

La escasa consciencia de desigualdad permea las prácticas cotidianas de trabajo doméstico, dificultando la negociación, y, con ello, un ejercicio efectivo de corresponsabilidad. Ello contribuye a la persistencia de prácticas asimétricas, de acuerdo a los recursos de poder diferenciado en la relación. En este sentido, cobra relevancia señalar los hallazgos del estudio sobre trabajo doméstico y de 
cuidados comparativo entre Chile-Argentina y España, de Domínguez, Rubilar y Muñiz (2019), que indican que en el caso Chileno la brecha de género se explicaría principalmente por elementos contextuales y por una ideología de género evidentemente desigualitaria, que condiciona la división sexual del trabajo.

Creemos que se han logrado avances, pero que estos aún no han llegado al nivel profundo que se requiere para lograr la igualdad de género efectiva. Resultaría interesante en futuros estudios explorar si estas lógicas de binariedad se reproducen en otros tipos de relaciones de pareja, como, por ejemplo, parejas del mismo sexo, ya que, si bien no se da la polaridad femenino-masculino, podrían surgir otras formas de relación de poder, como el NSE, que podrían seguir reproduciendo lógicas de desigualdad que impidan la corresponsabilidad en la esfera de lo doméstico.

Este estudio avanza en profundizar la comprensión sobre las transformaciones efectivas en los roles tradicionales de género, ya que, a pesar de veinte años de discurso público de igualdad de género, aún estos discursos no han logrado permear las prácticas concretas de negociación y distribución de trabajo doméstico y de cuidados en la cotidianeidad de los sujetos. Queda mucho por avanzar en la corresponsabilidad en el trabajo reproductivo, y comprender que, solo cuando se lo entienda como una contribución social de relevancia, es posible que logre un estatus que busque ser compartido.

Estudios, como el de Raquel Fernández (2013), señalan que a lo largo de las generaciones, observar a mujeres trabajar promovió que la mujer fuese normalizando su incorporación en el ámbito laboral. Podríamos pensar entonces que, aunque la participación de los hombres en lo doméstico aún es tímida, esta puede conllevar a cierta alteración cultural parecida.

No obstante, como se ha señalado en estas líneas, esos cambios no son tan simples. La forma en la que los hombres interactúan en la casa puede ser tramposa. Las labores domésticas, condicionadas por una lógica patriarcal, no suponen ningún logro significativo. No obstante, aprovechar esta situación para invitar a la reflexión de hombres y mujeres podría configurar un lúcido cambio social.

\section{Referencias}

Aguayo, F., Correa, P., y Cristi, P. (2011). Encuesta IMAGES Chile. Resultados de la encuesta internacional de masculinidades y equidad de género. Santiago: CulturaSalud/EME.

Arriagada, I. (2013). Desigualdades en la familia: trabajo y cuidado en Chile. En C. Mora (ed.), Desigualdad en Chile: la continua relevancia del género (pp. 91-112). Santiago: Ediciones Universidad Alberto Hurtado.

Arriagada, I., y Todaro, R. (2012). Cadenas globales de cuidados: el papel de las migrantes peruanas en la provisión de cuidados en Chile. Santiago: ONU Mujeres.

Candela, P., y Piñón, J. (2013). Vida, Trabajo y relaciones de género en la metrópolis global. [Madrid/Catarata]

Comunidad Mujer (2012). Boletin Mujer y Trabajo: Proyecto para ampliar la provisión de sala cuna, los nudos del debate. Serie comunidad Mujer no 17 . Recuperado el 17 de marzo de 2013, de: http://www.comunidadmujer.cl/estudios/series-de-estud $\operatorname{ios} / 3 /$ 
Chinchilla, N., Poelmans, S., y León, C. (2003). Politicas de conciliación trabajo-familia en 150 empresas españolas. Extraído el 18 de Mayo de 2007 desde http://www.ie se.edu/research/pdfs/DI-0498.pdf

Debeljuh, P., y Jáuregui, K. (2004). Trabajo y Familia: Hacia una cultura familiar amigable en el contexto latinoamericano. Esan-cuadernos de difusión, 16, 91-102.

Díaz Martínez, C., y Simó Noguera, C. (coord) (2016). La brecha salarial y la brecha de cuidados: Dos factores de desigualdad de género. Valencia: Tirant Humanidades.

Díaz Martínez, C., Dema Moreno, S., y Finkel, L. (2015). Desigualdades de género en la distribución de recursos económicos en las parejas españolas. Papers, 100(1), 53-73.

Domínguez, M., Muñiz, T., y Rubilar, G. (2019). El trabajo doméstico y de cuidados en las parejas de doble ingreso. Análisis comparativo entre España, Argentina y Chile. Papers, $104(2)$.

Doña, K. (2007). Estrategias para compatibilizar trabajo y familia en mujeres directivas del sector público chileno: estudio de caso. Recuperado el 20 de agosto de 2009, de: http://www.clad.org.ve/fulltext/0057525.pdf.

Durán, M. A. (2018). La riqueza invisible del cuidado. Universidad de Valencia.

ENPI. (2010). Encuesta Nacional de la Primera Infancia, ENPI 2010 - Resultados Preliminares. Santiago: JUNI/UNESCO/Unicef.

Farkas, C. (2008). Escala de evaluación parental (EEP): Desarrollo, propiedades psicométricas y aplicaciones. Universitas Psychologica, 7(2), 457-467.

Fernandez, R. (2013). Cultural Change as Learning: The Evolution of Female Labor Force Participation over a Century. American economy review. [103/1/476-478]

Ffrench-Davis, R. (2001). Entre el neoliberalismo y el crecimiento con equidad: tres décadas de politica económica en Chile. Santiago: Dolmen Ediciones.

Finkel, L. (1997). El Reparto del Trabajo Doméstico en la Familia: La Socialización en las Diferencias de Género. Madrid: Confederación Española de Asociaciones de Madres y Padres de Alumnos (CEAPA) e Instituto de la Mujer.

Fundación Chile Unido (2013). Estudio mejores empresas para padres y madres que trabajan. Informe General 2013. Recuperado el 7 de mayo de 2014, de: http://www.chileunido.cl/wp-content/uploads/2014/01/Informe-GeneralEstudio-Mejores-Empresas-2013.pdf

Gornick, J. \& Meyers, M. (2009). Institutions that support gender equality in parenthood and employment. En J. Gornick \& M. Meyers (eds.), Gender equality: transforming family divisions of labor (pp. 3-66). Londres: Verso.

INE-Chile Instituto Nacional de Estadísticas (2016a). ENUT Encuesta Nacional Sobre Uso del Tiempo. Documento Metodológico. Santiago de Chile: INE.

INE-Chile Instituto Nacional de Estadísticas (2016b). ENUT Encuesta Nacional Sobre Uso del Tiempo. La Dimensión Personal del Tiempo, ENUT 2015. Santiago de Chile: INE.

Jiménez, A., y Moyano, E. (2008). Factores laborales de equilibrio entre trabajo y familia: medios para mejorar la calidad de vida. Revista Universum, 23(1), 116-133.

JUNJI/UNESCO/Unicef (2010). Encuesta Nacional de la Primera Infancia, ENPI 2010 - Resultados Preliminares. Santiago: JUNI/UNESCO/Unicef. Recuperado el 12 de diciembre de 2013, de: http://web.integra.cl/doctos_cedoc/archivos/do cumentos/Informe\%20Final\%20Preliminar\%20ENPI.pdf

Hooks, B. (2017). El feminismo es para todo el mundo. Traficantes de sueños, Mapa. 
Lamaute-Brisson, N. (2013). Redistribuir el cuidado: para un nexo de políticas públicas. En C. Calderón (coord.), Redistribuir el cuidado. El desafío de las políticas (pp. 69-126). Santiago de Chile: CEPAL.

LYD. (2006). Mujer: Politicas de Conciliar Familia y Trabajo. Recuperado el 10 de agosto de 2010, de: http://www.lyd.com/lyd/controls/neochannels/neo_ch380 6/deploy/tp761mujer\%20politicas.

Martínez Franzoni, J. (2008). ¿Arañando bienestar? Trabajo remunerado, protección social y familias en América Central. Buenos Aires: Consejo Latinoamericano de Ciencias Sociales. Recuperado el 13 de agosto de 2011, de: http://bibliotecavirtu al.clacso.org.ar/ar/libros/clacso/crop/franzoni/05Cap3.pdf

Ministerio de Desarrollo Social (2017) Encuesta CASEN 2017, módulo Educación, Trabajo, Infancia, Equidad de género.

Ministerio de Desarrollo Social / Ministerio de Salud (2011) Cuatro años creciendo juntos: Memoria de la instalación del Sistema de Protección Integral a la Infancia ChileCrece Contigo, 2006-2010. Recuperado el 15 de mayo de 2012, de: http://www.crececontigo.gob.cl/wpcontent/uploads/2013/05/ChCC_ME MORIA.pdf.

OECD (2011). Doing better for families. Washington: OECD Publishing.

Organización Internacional del Trabajo (2018). Perspectivas sociales y del empleo en el mundo. Mujeres. Tendencia del empleo femenino 2018.

OIT \& PNUD (2009). Trabajo y Familia: Hacia nuevas formas de conciliación con corresponsabilidad social. Santiago: OIT y PNUD. Recuperado el 22 de septiembre de 201, de: http://www.oit.org.pe/WDMS/bib/publ/...trab_familia\%5BOITP NUD\%5D.pdf

Olavarría, J. (2004). ¿Dónde está el nuevo padre? De la retórica a la práctica. En X. Valdés y T. Valdés (eds.), Familia y vida privada: ¿transformaciones, resistencias o nuevos sentidos? (pp. 215-250). Santiago: CEDEM/FLACSO.

Otárola, G. (2007). La relación existente entre el conflicto trabajo- familia y el estrés individual en dos organizaciones colombianas. 3, 20(34), 139- 160.

Pautassi, L. (2010). Cuidado y Derechos: la nueva cuestión social. En S. Montaño y C. Calderón (eds.), El cuidado en acción: entre el derecho y el trabajo (pp. 69-92). Santiago: CEPAL/AECID/UNIFEM.

Pérez, G. (2004). Investigación cualitativa: Retos e interrogantes. Madrid: Editorial La Muralla.

Prieto C., (coord.) (2015). Trabajo, cuidados, tiempo libre y relaciones de género en la sociedad española. Madrid, Ediciones CINCA.

Programa de las Naciones Unidas para el Desarrollo (PNUD). (2010) Género: los desafios de la igualdad. Santiago de Chile: PNUD.

Razavi, S. (2007). The political and social economy of care in a Development context: contextual issues, research questions, and policy options. Ginebra: Instituto de Investigación de Desarrollo Social, ONU. Recuperado el 7 de septiembre de 2009, de: http://www.unrisd.org/80256B3C005BCCF9/\%28httpAuxPages\%2 9/2DBE6A93350A7783C12573240036D5A0/\$file/Razavi-paper.pdf

Román, J., Avendaño., C., y Tomicic, A. (2004). Conflicto y equilibrio en la relación trabajo familia: Un estudio con mujeres trabajadoras por cuenta propia. Persona y Sociedad, 18(1), 51-76.

Saldaña, L. (2018). Relaciones de género y arreglos domésticos: Masculinidades cambiantes en Concepción, Chile. Polis (Santiago),17(50),183-204. 
Schkolnik, M. (2004) ¿Por qué es tan increíblemente baja la tasa de participación de las mujeresen Chile? Documento En Foco No 33. Recuperado el 14 de marzo de 2008, de. http://www.expansiva.cl/media/en_foco/documentos/06102004132738.pd f.

Selamé, T. (2004). Mujeres, brechas de equidad y mercado de trabajo: Chile. Proyecto Ge\#nero, Pobreza y Empleo. Santiago: OIT/PNUD.

SERNAM (2002). Conciliación entre la vida laboral y la vida familiar de trabajadores y trabajadoras chilenos/as: Documento de trabajo $\mathrm{N}^{\circ} 76$. Recuperado el 18 de mayo de 2007, de: http://www.sernam.cl/

Servicio Nacional de la Mujer (2012). Estudio sobre las principales preocupaciones y anhelos de las madres de hoy en Chile - Informe final de resultados. Santiago: SERNAM/Demoscopica.

Sojo, A. (2011). De la evanescencia a la mira: El cuidado como eje de politicas y de actores en América Latina. Serie Seminarios y Conferencias No 67. Santiago de Chile: CEPAL.

Servicio Nacional de la Mujer (2002). Conciliación entre la vida laboral y la vida familiar de trabajadores y trabajadoras chilenos/as. Departamento de Estudios y Estadísticas, $n^{\circ}$ 76. Santiago: SERNAM.

Tobío, C. (2002). Conciliación o contradicción: Cómo hacen las madres trabajadoras. Revista Española de Investigaciones Sociológicas, 97, 155-186.

Torío, S., Peña, J., Rodríguez, M., Fernández, M., y Molina, S. (2010). Hacia la corresponsabilidad familiar: Construir lo cotidiano. Un programa de educación parental. Education Siglo XXI, 28(1), 85-108.

Undurraga, R. (2013). Mujer y trabajo en Chile: ¿qué dicen las mujeres sobre su participación en el mercado laboral? En C. Mora (ed.), Desigualdad en Chile: la continua relevancia del género (pp. 113-141). Santiago: Ediciones Universidad Alberto Hurtado.

Wengraf, T. (2004). Qualitative research interviewing: biographic narrative and semistructured methods. Londres: SAGE

\section{Notas}

1 Este trabajo ha sido realizado en el marco del programa de doctorado interuniversitario en Estudios de Género: Culturas, Sociedades y Políticas. Universidad de Barcelona. Este artículo se ha elaborado en el contexto de la Red INCASI, proyecto europeo que ha recibido financiación del programa de investigación H2020 de la Unión Europea, Marie Skłodowska-Curie GA No 691004, coordinado por el Dr. Pedro López-Roldán. El artículo refleja la opinión de los autores y la Agencia no es responsable del uso que se pueda hacer de la información que contiene.

2 Nos referimos a la Ley No 20.047, que favorece a los padres en el contexto del nacimiento de sus hijos e hijas, cubre el espectro tanto público como privado, posee un carácter irrenunciable, y sus costos recaen sobre el empleado.

3 Aquellas creencias personales sobre las propias capacidades para lograr ser un buen padre. Todo esto trae consigo tener expectativas reales y además ser capaces de percibirse a sí mismos como padres competentes. 